\title{
Lagging chromosomes entrapped in micronuclei are not 'lost' by cells
}

Cell Research (2012) 22:932-935. doi:10.1038/cr.2012.26; published online 21 February 2012

\section{Dear Editor,}

In the literature, chromosomes lagging during anaphase and entrapped in micronuclei are considered to be a sign of genetic material loss and closely associated with aneuploidy formation [1-4]. However, some of these assumptions were based on analysis of fixed cells, and the actual behaviors of lagging chromosomes and micronuclei in live cells are poorly characterized [5]. To avoid the shortcomings associated with fixed samples, we previously labeled all the chromosomes with fluorescent protein for live cell imaging and have provided preliminary data on the formations of lagging chromosomes, micronuclei and their effect on the fate of cells $[6$, 7]. Unfortunately, labeling of all chromosomes prevents us from precisely tracking the fates of lagging chromosomes and micronuclei [7]. To address these issues, new experimental strategies are required.

In this study, colorectal cancer HCT116 cells were used to examine the fates of cells containing lagging chromosomes or micronuclei. We generated derivative clones with all the chromosomes labeled with red fluorescent protein (by expression of the mCherry-human histone $2 \mathrm{~B}$ fusion gene) and one chromosome labeled with green fluorescent protein (GFP), which is achieved by integrating the Escherichia coli Lac operator (LacO) sequence into a single chromosomal locus in cells expressing the GFP-Lac repressor (LacI) fusion gene (Supplementary information, Data S1). Thus, the localization and dynamics of the single GFP-tagged chromosomes in every phase of cell cycle could be monitored in live cells (Figure 1Aa). Three clones that contain one copy of the GFP-labeled chromosome (4, 6 or 15$)$ were chosen for further analysis. They displayed a karyotype, cell fates and cell cycle duration indistinguishable from the parental HCT116 cells (Supplementary information, Figures $\mathrm{S} 1, \mathrm{~S} 2$ and Table S1).

Merotelic kinetochore (single kinetochore attaches to microtubules from two spindle poles) has been proposed to be the major mechanism underlying the spontaneous formation of lagging chromosomes, which can be remarkably enhanced in cells released from nocodazole treatment [8]. To examine the fates of lagging chromosomes, we utilized nocodazole treatment to increase the incidence of the GFP-tagged laggards (Supplementary information, Figure S3) and employed live cell imaging to track their fates (Supplementary information, Figure S4). We found that vast majority of the lagging chromosomes $(84.62 \%-93.33 \%)$ eventually moved to the correct side of spindle. They often ended up as micronuclei in the daughter cells. Infrequently $(6.67 \%-15.38 \%)$, the laggards ultimately moved to the incorrect side and produced a trisomic daughter cell with the laggard in micronucleus and a monosomic cell. Consistently, it has been indicated that micronucleus was more likely to reside in the correct cells [9]. Thus, most lagging chromosomes do not directly produce aneuploid progenies, but are entrapped in the micronuclei of correct daughter cells.

Given that the abnormal chromosome number often causes a proliferative disadvantage [10], we next explored whether chromosomes entrapped in micronuclei can also affect cell proliferation. To resolve this issue, we used long-term live cell imaging to estimate the proliferation potential of the cells containing GFP-tagged micronuclei generated from nocodazole-induced segregation error (Figure 1A and 1B, Supplementary information, Figure S5).

Nocodazole-treated mitotic cells were collected by shake-off and washed out. Cells were then plated for $8 \mathrm{~h}$ to permit the completion of mitosis prior to $24 \mathrm{~h}$ of timelapse imaging. According to the descendents generated from anaphase cells harboring GFP-tagged laggards (Supplementary information, Figure S4), four types of daughter cells were tracked: (i) cells containing one GFP-tagged chromosome in nuclei (Figure 1Aa); (ii) or in micronuclei (Figure 1Ab, left-side cell); (iii) cells containing two GFP-tagged chromosomes, one each in the main nucleus and in the micronucleus (Figure 1Ac, rightside cell); and (iv) cells lacking the GFP-tagged chromosomes (Figure 1Ac, left-side cell).

For the untreated cells, only those containing one GFP-tagged chromosome in nuclei (scenario i) were ob- 
served, of which $96.00 \%-97.00 \%$ entered mitosis during the $24 \mathrm{~h}$ of tracking (Figure 1B and Supplementary information, Figure S5). However, for the daughter cells generated from mitotic cells that released from nocodazole treatment, the frequencies (scenario i) of entering mitosis were decreased to $70.79 \%-76.30 \%$, with the remaining cells undergoing cell cycle arrest or apoptosis (Figure $1 \mathrm{~B}$ and Supplementary information, Figure S5). These can be explained by the fact that missegregation of unlabeled chromosomes causes a p53-induced proliferative disadvantage [10]. Interestingly, similar frequencies were observed in cells containing one GFP-tagged chromosome in micronuclei (scenario ii), with 67.65\%-73.33\% of the micronucleus-bearing cells entering mitosis (Figure $1 \mathrm{Ab}$ and $1 \mathrm{~B}$, Supplementary information, Figure S5). These results argue that chromosomes entrapped in micronuclei do not substantially affect the proliferation of the cells containing them. Conversely, if the cells gained (scenario iii) or lost a copy of chromosomes (scenario iv), their proliferation were significantly compromised with $57.89 \%-91.67 \%$ of the cells undergoing cell cycle arrest or apoptosis (Figure 1Ac and 1B, Supplementary information, Figure S5). In addition, loss of a chromosome (scenario iv) is more detrimental to cells than gain of a chromosome (scenario iii) (Figure 1B and Supplementary information, Figure S5). Therefore, gain or loss of chromosomes causes a proliferative disadvantage, but chromosomes entrapped in micronuclei per se do not impair the proliferation of the cells containing them.

These results provide ample evidence that maintaining the integrity of chromosome composition of a cell is crucial for cell survival and proliferation, no matter whether a chromosome is present in the main nucleus or micronucleus. Thus, chromosomes entrapped in micronuclei are not ignored by their host cells regarding pathways involved in cell fate determination. Previously, we have found that cells with micronuclei are more likely to die or undergo cell cycle arrest when compared with the micronucleus-free counterparts [7]. Combining the present findings with the previous ones, it is possible that such detrimental effects were only exerted by micronuclei originated from missegregated or damaged chromosomes but not by micronuclei generated from correctly segregated chromosomes.

Finally, we examined the destinies of micronuclei in cycling cells. Possible scenarios for the fates of the micronuclei include: (1) expulsion from the cell [3]; (2) apoptosis independent of the cell [2]; (3) removal by autophagy [4]; (4) retention within the cytoplasm as an extra-nuclear entity despite cell division [6]; or (5) reincorporation into the main nucleus [9]. The first four scenarios would cause genetic material loss from the cell. However, some of these putative destinies of the micronuclei were confusing because the results were based on analysis of fixed cells and it was difficult to verify in live cells with all chromosomes labeled. For example, although micronuclei might disappear in cells, it was hard to determine whether they were degraded, eliminated or overlaid with the main nuclei [7]. Fortunately, using the cells with a single chromosome labeled with GFP, the micronuclei harboring the labeled chromosomes can be followed as distinct fluorescent dots during cell division, and thus the destinies of micronuclei can be determined.

We scrutinized the behaviors of micronuclei containing GFP-labeled chromatins following nocodazole treatment (Figure 1C and 1D). After analyzing time-lapse videos of 182 micronuclei each with a GFP dot in 182 cells, we did not observe micronuclei expulsion from the cells or degradation in situ. These imply that the genetic materials in micronuclei are not lost by the cells. Importantly, when micronucleus-bearing cells entered mitosis, almost all of the GFP-labeled chromatins encapsulated in micronuclei $(97.03 \%$, or $98 / 101)$ were able to condense, congregate onto metaphase plate, separate and segregate into two daughter nuclei normally as the chromosomes in the main nuclei, regardless of the micronuclei in the correct (Figure $1 \mathrm{Ab}$ and $1 \mathrm{Ca}$ ) or incorrect (Figure $1 \mathrm{Cb}$ ) cells. Subsequently, such divisions produced two daughter cells without micronuclei (summarized in Figure 1D). Only 3 out of 101 GFP-tagged micronuclei remained as an independent entity during cell divisions (Figure $1 \mathrm{Cc}$ and 1D). The chromatins in these micronuclei did not appear to replicate because the GFP dots did not double after division. Given that the genetic material of these micronuclei did not join the chromosomal mass of the main nuclei during mitosis, we can speculate that these micronuclei lack a functional centromere. Indeed, both anaphase laggards and micronuclei composed of acentric chromosome fragments were occasionally observed at a similar frequency (Supplementary information, Figure S6).

Collectively, these results demonstrate that the chromosomes encapsulated in micronuclei behave normally as those in the main nuclei during cell division, and subsequently lead to the formation of cells without micronuclei. Therefore, the commonly used "loss" as a synonym for micronucleation is not accurate [1], at least in this type of micronuclei (originating from an intact chromosome), as the micronuclei appear to be functional. However, it is noteworthy that micronuclei can also originate from extrachromosomal element, namely, double minute (DM) in some cell lines. The origins and destinies of the DM-type micronuclei are far different from the chromosome-type micronuclei, and the DM-type micronuclei 

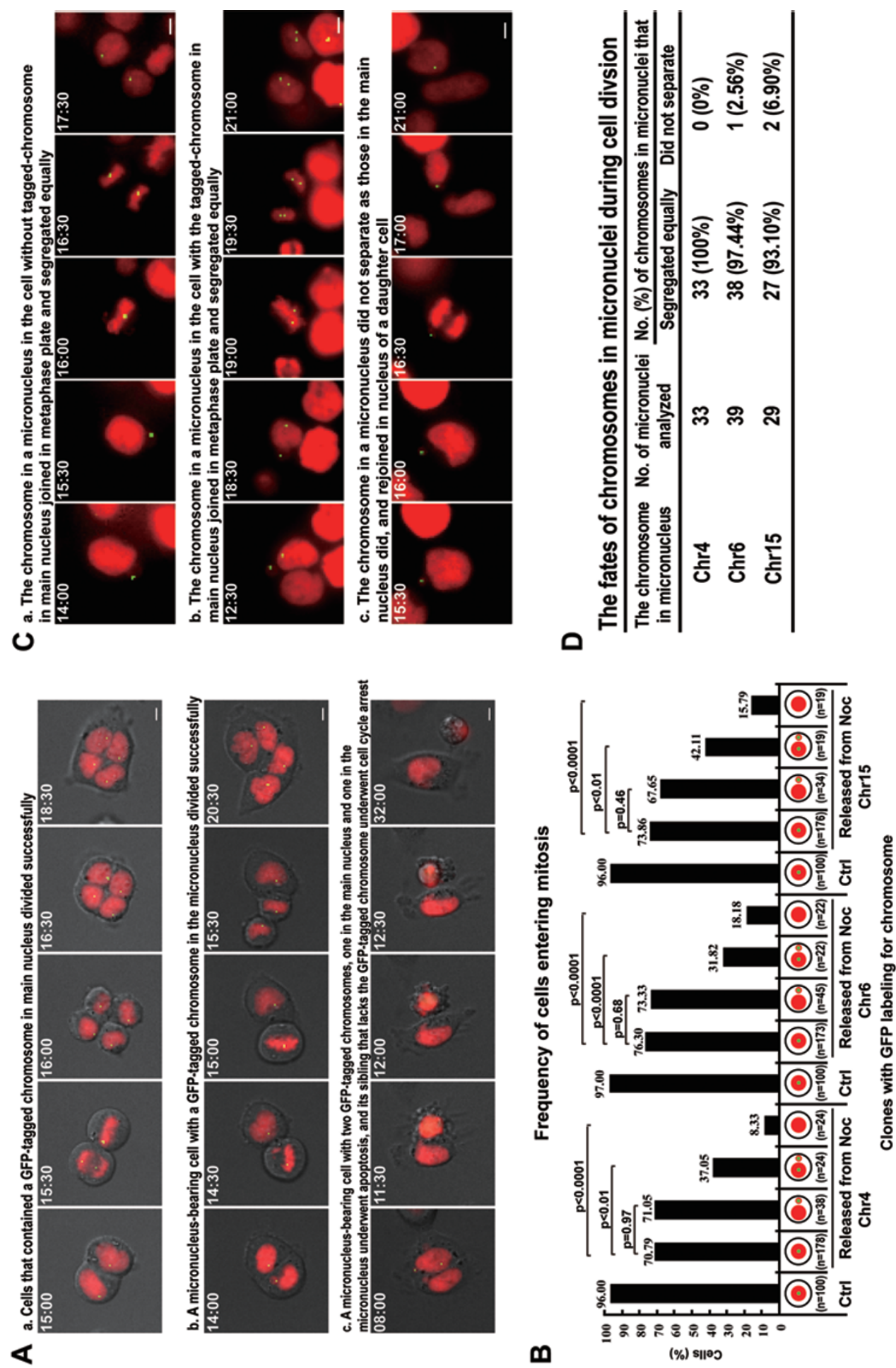

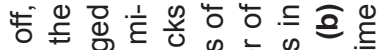

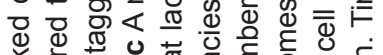

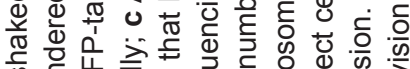
क 凹 ब ऐ)

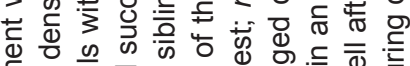

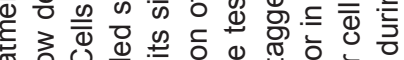

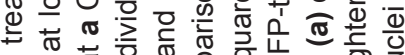
ब 䨌

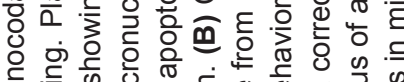

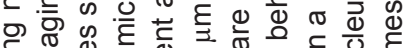
ᄃ ब. ठ

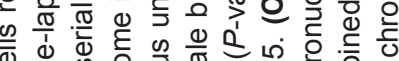
क U 는 Ð

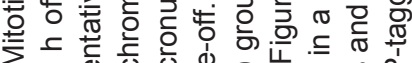

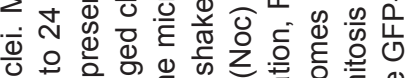

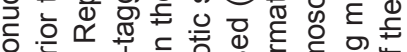

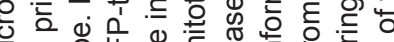
ह $\frac{\infty}{\omega}$ 융

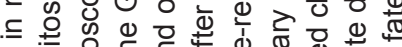

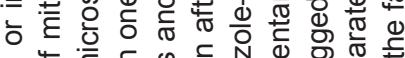
ब 들 응

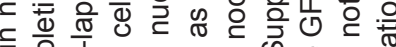
है है

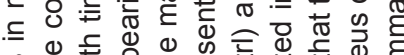

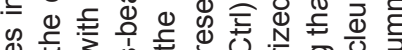

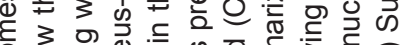
రิ

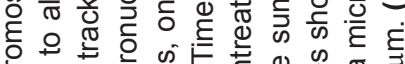
은 인 क ᄃ ᄃ

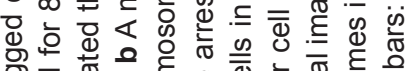
宁

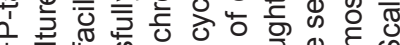
U

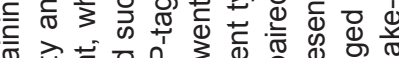

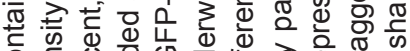

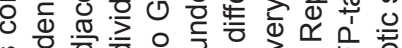

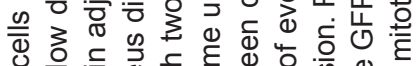

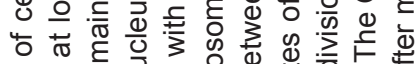

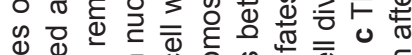

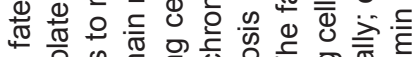

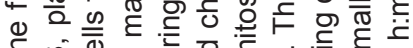
F⿻

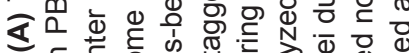

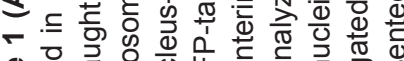

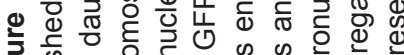

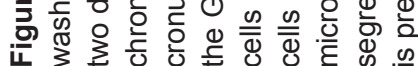


can be extruded from the cells via cytoplasmic membrane blebbling [3].

In conclusion, our results directly demonstrate that most of the lagging whole chromosomes do not induce aneuploidy but reside in the correct daughter cells as micronuclei. The chromosomes entrapped in micronuclei do not substantially affect the proliferation of the host cells. They behave normally as those in main nuclei during cell division. Thus, the lagging chromosomes entrapped in micronuclei are not 'lost' by cells. Although these findings were obtained from one cell line, they were shared among three clones with a different chromosome labeled in each clone, indicating that the observed phenomena were generally applicable to different chromosomes. Further studies on whether these findings are applicable to other cells and exist in vivo as well are still required.

\section{Acknowledgments}

We thank Drs Chenbei Chang (University of Alabama at Birmingham, USA), Steffen Dietzel (Ludwig-Maximilians-Universität München, Germany), Wendy A Bickmore (University of Edinburgh, UK), Mingrong Wang and Shujun Cheng (Chinese Academy of Medical Sciences and Peking Union Medical College) for providing plasmids. This work was supported by the National Natural Science Foundation of China (30671168 and 30725013) and Doctoral Fund of Ministry of Education of China (20070358022).

Yun Huang ${ }^{1}$, Long Jiang ${ }^{1}$, Qiyi $\mathrm{Yi}^{1}$, Lei Lv ${ }^{1}$, Zheng Wang ${ }^{1}$, Xiaoyu Zhao ${ }^{1}$, Liangwen Zhong ${ }^{1}$, Hanwei Jiang ${ }^{1}$, Salma Rasool ${ }^{1}$, Qiaomei Hao ${ }^{1}$, Zongyou Guo ${ }^{2}$, Howard J Cooke ${ }^{1,3}$, Michael Fenech ${ }^{4}$, Qinghua $\mathrm{Shi}^{1}$

${ }^{I}$ Hefei National Laboratory for Physical Sciences at Microscale and School of Life Sciences, University of Science and Technology of China, Hefei, Anhui 230026, China; ${ }^{2}$ Department of Dermatology, Brigham and Women's Hospital and Harvard Skin Disease Research Center, Harvard Institutes of Medicine, Room 664, 77 Ave. Louis Pasteur, Boston, MA
02115, USA; ${ }^{3}$ MRC Human Genetics Unit and Institute of Genetics and Molecular Medicine, Western General Hospital, Edinburgh, UK; ${ }^{4}$ CSIRO Food and Nutritional Sciences, PO Box 10041, Adelaide BC, Adelaide, SA 5000, Australia

Correspondence: Qinghua Shi

Tel: +8605513600344

E-mail: qshi@ustc.edu.cn

\section{References}

1 Ford JH, Schultz CJ, Correll AT. Chromosome elimination in micronuclei: a common cause of hypoploidy. Am J Hum Genet 1988; 43:733-740.

2 Gernand D, Rutten T, Varshney A, et al. Uniparental chromosome elimination at mitosis and interphase in wheat and pearl millet crosses involves micronucleus formation, progressive heterochromatinization, and DNA fragmentation. Plant Cell 2005; 17:2431-2438

3 Utani K, Okamoto A, Shimizu N. Generation of micronuclei during interphase by coupling between cytoplasmic membrane blebbing and nuclear budding. PLoS One 2011; 6:e27233.

4 Rello-Varona S, Lissa D, Shen S, et al. Autophagic removal of micronuclei. Cell Cycle 2012; 11:170-176.

5 Terradas M, Martin M, Tusell L, Genesca A. Genetic activities in micronuclei: is the DNA entrapped in micronuclei lost for the cell? Mutat Res 2010; 705:60-67.

6 Rao X, Zhang Y, Yi Q, et al. Multiple origins of spontaneously arising micronuclei in HeLa cells: direct evidence from longterm live cell imaging. Mutat Res 2008; 646:41-49.

7 Huang Y, Hou H, Yi Q, et al. The fate of micronucleated cells post X-irradiation detected by live cell imaging. DNA Repair (Amst) 2011; 10:629-638.

8 Cimini D, Howell B, Maddox P, Khodjakov A, Degrassi F, Salmon ED. Merotelic kinetochore orientation is a major mechanism of aneuploidy in mitotic mammalian tissue cells. $J$ Cell Biol 2001; 153:517-527.

9 Thompson SL, Compton DA. Chromosome missegregation in human cells arises through specific types of kinetochoremicrotubule attachment errors. Proc Natl Acad Sci USA 2011; 108:17974-17978.

10 Thompson SL, Compton DA. Proliferation of aneuploid human cells is limited by a p53-dependent mechanism. $J$ Cell Biol 2010; 188:369-381.

(Supplementary information is linked to the online version of the paper on the Cell Research website.) 